Bristol, Speakers bureau: Abbvie, Pfizer, Roberta Priori: None declared Alessia Alunno: None declared, Guido Valesini: None declared, Roberto Giacomelli Grant/research support from: Actelion, Pfizer, Speakers bureau Abbvie, Roche, Actelion, BMS, MSD, Ely Lilly, SOBI, Pfizer, Roberto Gerli: None declared, Chiara Baldini: None declared, Athanasios Tzioufas: None declared, Salvatore De Vita Consultant of: Roche, Human Genome Science, Glaxo Smith Kline and Novartis

DOI: 10.1136/annrheumdis-2020-eular.3689

\section{THU0266 RESILIENCE IN WOMEN WITH PRIMARY SJÖGREN'S SYNDROME}

F. Giardina ${ }^{1}$, G. Curcio ${ }^{2}$, R. Izzo ${ }^{1}$, S. Colafrancesco ${ }^{1}$, A. Gattamelata ${ }^{1}$ L. Mastromanno ${ }^{1}$, G. Valesini ${ }^{1}$, R. Priori ${ }^{1}$. ${ }^{1}$ Sapienza Università di Roma, UOC Reumatologia, Dipartimento di Scienze Cliniche, Internistiche, Anestesiologiche e Cardiovascolari, Roma, Italy; ${ }^{2}$ Università degli Studi dell'Aquila, Dipartimento di Scienze Cliniche Applicate e Biotecnologiche, L'Aquila, Italy

Background: Resilience is the ability to react positively to stressful life events, a multidimensional feature that varies in relation to context, time, age, sex, culture and personal experience, appearing among the most important traits in patients suffering from rheumatic diseases ${ }^{1}$. Several studies focus on patients with RA and SLE and the role of resilience in the respective clinical manifestations, as well as in the development of anxiety and depression ${ }^{2,3}$. Conversely, the data available regarding patients with primary Sjögren's Syndrome (pSS) are limited. Objectives: To assess, in women with pSS (classified according to the criteria of Vitali et al. ${ }^{4}$ ), the relationship between resilience and anxiety, depression, health, fatigue, physical activity and quality of life in relation to disease activity and duration and in consideration of demographic, job and cultural characteristics.

Methods: 74 female patients with pSS afferent to the dedicated clinic of the University Hospital Policlinico Umberto I of Rome were recruited. Resilience was assessed by administering the Italian validated version of the Resilience Scale (RS-14) $)^{5}$ consisting of 14 items, each of which is assigned a score from 1 to 7 , with a range from 14 to 98 . Higher scores relate to greater resilience. ESSDAI (EULAR Sjögren's syndrome disease activity index), ESSPRI (EULAR Sjogren's Syndrome Patient Reported Index), SSDDI (Sjogren's Syndrome Disease Damage Index) were assessed and EuroQol / GH EQ VAS (visual analogue scale), HADS (Hospital Anxiety and Depression Scale), SF-12 (Short-form 12 health survey), FAS (Fatigue Assesment Scale), IPAQ (International Physical Activity Questionnaire), FACIT-F (Functional Assessment of Chronic Illness Therapy - Fatigue) questionnaires were submitted. Educational qualifications and job were also considered. The statistical analysis was carried out by means of Spearman's correlation.

Results: No relationship was found between resilience, systemic disease activity, disease duration, patient-reported symptoms and damage. Furthermore, no apparent link was found between socio-demographic characteristics, employment and resilience. Conversely, an inverse relationship was found between resilience and mood disorders $(p=0.0379)$, with greater resilience associated with a better perception of quality of life $(p=0.0232)$ and general health $(p=0.0002)$, mainly mental $(p=0.0001)$ than physical $(p=0.0035)$, as well as less fatigue $(p=0.0079)$ and more phyisically active lifestyle $(p=0.0012)$

Conclusion: For the first time, the role of resilience in women with pSS in relation to their disease and other individual parameters was assessed. The most resilient patients are less depressed and show better perception of their health. Greater resilience tends to correlate with less anxiety, physical and mental fatigue and a more active lyfestile, while there was no relation between resilience value, active disease and socio-demographic features.

References:

[1] Rojas M. et al., Resilience in women with autoimmune rheumatic diseases, Joint Bone Spine (2017)

[2] Sílvia Fernanda Cal et al., Resilience in systemic lupus erythematosus, Psychology, Health \& Medicine (2013), 18:5, 558-563.

[3] NeiLi Xu et al., Associations of perceived social support and positive psychological resources with fatigue symptom in patients with rheumatoid arthritis. PLoS One. 2017; 12(3).

[4] Vitali C, et al. Classification criteria for Sjogren's syndrome: a revised version of the European criteria proposed by the American-European Consensus Group. Ann Rheum Dis 2002; 61:554-558.

[5] Camilla Callegari et al., Reliability and validity of the Italian version of the 14-item Resilience Scale, Psychology Research and Behavior Management 2016:9 277-284

Disclosure of Interests: None declared

DOI: 10.1136/annrheumdis-2020-eular.4757

\section{THU0267 \\ SERUM IGG2 LEVELS PREDICT VERY LONG-TERM PROTECTION FOLLOWING PNEUMOCOCCAL VACCINE IN SYSTEMIC LUPUS ERYTHEMATOSUS (SLE)}

T. Goulenok ${ }^{1}$, A. L. Gerard ${ }^{1}$, M. Bahuaud ${ }^{2}$, P. Aucouturier ${ }^{3}$, H. Moins ${ }^{1}$, T. Papo ${ }^{1}$, K. Sacre ${ }^{1} .{ }^{1}$ Université Paris Diderot, Paris, France; ${ }^{2}$ Université
Paris Descartes, Paris, France; ${ }^{3}$ Université Pierre et Marie Curie, Paris, France

Background: Systemic lupus erythematosus (SLE) patients are at increased risk for Streptococcus pneumoniae infection. Although pneumococcal vaccination is an attractive method to prevent invasive pneumococcal infection vaccination coverage remains dramatically low in SLE. Moreover, the efficacy of vaccination may be reduced in SLE patients and sequential pneumococca vaccination using new conjugated pneumococcal vaccines in combination with 23-valent pneumococcal polysaccharide vaccine (PPV23) is now advocated However, limited study directly addressed the immune efficacy of such primeand-boost strategy in SLE

Objectives: We aimed to measure the immunological efficacy of the sequential pneumococcal vaccination using PCV13 in combination with PPV23 and identify factors associated with long-term immune protection following vaccination in SLE.

Methods: SLE patients received PCV13 vaccine followed by PPSV23 vaccine 8 weeks later. Immune protection, defined by an antigen-specific IgG concentration $\geq 1.3 \mu \mathrm{g} / \mathrm{mL}$ for at least $70 \%$ of 7 pneumococcal serotypes $(4,6 \mathrm{~B}, 9 \mathrm{~V}, 14,18 \mathrm{C}$, 19F, 23F), was assessed at baseline, 2 months, 12 months, and 36 months, defining very long-term protection.

Results: 21 (40[25-75] years; 85.7\% female) SLE patients received the sequential PCV13/PPV23 vaccines. Only 10 (47.6\%) showed a sustained immune protection against pneumococcal infection 36 months after PCV13 shot (very long-term protected, VLTP). Eleven patients had no long-term protection (NLTP) with a seroconversion that never $(n=6)$ or only transiently $(n=5)$ occurred. SLE disease features, treatment received and immunological characteristic did not differ between VLTP and NLTP patients except for a lower serum IgG2 levels in $\operatorname{NLTP}(1.45$ [1.30, 1.82] vs 3.30 [2.92, 4.44] $\mathrm{g} / \mathrm{L}, \mathrm{p}<0.001)$. Noteworthy the ROC curve showed that the serum IgG2 level before vaccination (AUC 0.95 [95\% Cl: 0.84-1]; $p=0.004$ ) was predictive for very long-term protection. A baseline serum IgG2 level of $2.125 \mu \mathrm{g} / \mathrm{ml}$ or more showed a sensitivity of $100 \%$ and a specificity of $90.9 \%$ for very longterm protection

Conclusion: The benefit of sequential PCV13/PPV23 vaccination in SLE is limited. Baseline IgG2 serum level before vaccination is strongly indicative of very long term protection following vaccination

Disclosure of Interests: None declared

DOI: 10.1136/annrheumdis-2020-eular.783

\section{THU0268 NEURO-DEVELOPMENTAL OUTCOME IN CHILDREN BORN TO MOTHERS WITH SLE AND APS}

M. Hassanien ${ }^{1}$, E. Talaat ${ }^{1}$, H. Abdellatif ${ }^{2} .{ }^{1}$ Assuit University, Assuit, Egypt; ${ }^{2}$ Aswan University, Assuit, Egypt

Background: Systemic Lupus erythematosus and antiphospholipid disease are very common autoimmune diseases in women at reproductive age.

Objectives: Evaluate the neuro-developmental outcome in children born to mothers with SLE or APS and to assess and characterize memory impairment in children's born to mother with systemic lupus erythematosus or APS using children's memory scale and the relation between tetrahydrobiopterin concentration range of children with developmental and neurologica disorders.

Methods: Women attending rheumatology clinics University of Asyut, SLE patients were eligible if they met the American College of Rheumatology (ACR) criteria for SLE and APL prior to pregnancy, and had at least one live birth following SLE diagnosis. Maternal history Data collected using a structured format that included medical and obstetric history. A detailed history of medication exposures and the Systemic Lupus Erythematosus Disease Activity Index (SLEDAI) during pregnancy was obtained. Offspring history Medical and developmental histories of the offspring including antenatal delivery, prenatal and pediatric histories, as child's cognitive, physical or social maturity compared with established age-appropriate norms. Speech or hearing delays, diagnosis of attention- deficit hyperactivity disorder (ADHD) or any special educational needs (eg, occupational or speech therapy, behavioral counseling) was recorded. Assessment and characterization of memory impairment using children's memory scale by neurologists. Tetrahydrobiopterin was measured by ELISA compared to children born to control healthy subjects of the same age and sex.

Results: Data on 38 mothers and 60 offspring were analysed: ADHD was reported for 15 of 60 (25\%) offspring. Recent memory delay was detected in $93 \%$ (14/15) Speech delay 40\% (6/15). Maternal APS history was significantly associated with increased use special educational need among offsprings, including after adjustment for lupus anticoagulant (LA) positivity $(39.4 \%$ for delays age $>2$ years; $p<0.05$ ). Anticardiolipin and anti-BETA2GP1 were not detected to be associated with delays. Recent memory delay was associated with increased Tetrahydrobiopterin level $(P=0.01)$. 